\title{
The VHL tumor suppressor protein regulates tumorigenicity of U87-derived glioma stem-like cells by inhibiting the JAK/STAT signaling pathway
}

\author{
HIROSHI KANNO ${ }^{1,2}$, HIDEMITSU SATO ${ }^{1}$, TAKA-AKIRA YOKOYAMA ${ }^{1}$, \\ TETSUYA YOSHIZUMI $^{1}$ and SACHIKO YAMADA ${ }^{1}$ \\ ${ }^{1}$ Department of Neurosurgery, Yokohama City University School of Medicine, Yokohama; \\ ${ }^{2}$ Department of Neurosurgery, Yokosuka City University, Yokosuka, Japan
}

Received November 8, 2012; Accepted December 17, 2012

DOI: $10.3892 /$ ijo.2013.1773

\begin{abstract}
The signal transducer and activator of transcription 3 (STAT3) factor plays an important role in the tumorigenicity of cancer stem cells. The purpose of this study was to investigate the inhibitory mechanism of this pathway acting through the tumor suppressor von Hippel-Lindau (VHL) protein in glioma cancer stem cells. We isolated floating neurosphere-forming $\mathrm{CD}_{133^{+}}$cells as glioma stem-like cells (GSLCs) by the MACS method. Furthermore, we examined these cells for their growth rate, ability to form colonies and neurospheres in soft agar, capacity for implantation into SCID mice and expression of CD133, STAT3, JAK2, Elongin A, PTEN and VHL. Furthermore, we transferred the VHL gene, an inhibitor of STAT3, into GSLCs using an adenovirus vector and compared these transfectants with control vector-transfected GSLCs. GSLCs proved to be implantable and formed a tumor in the subcutaneous tissue of SCID mice, the histology of which was similar to that of human glioblastomas. In addition, GSLCs exhibited a high capacity for soft agar colony and neurosphere formation, nearly all of which were CD133 positive. The majority of GSLCs were immunopositive for STAT3, JAK2 and Elongin A, but immunonegative for PTEN and VHL. When the VHL gene was transferred to GSLCs and these cells were transplanted into SCID mice, they did not result in tumor formation. Their capacity for soft agar colony and neurosphere formation was significantly inhibited, although their proliferation was only moderately inhibited. Regarding the expression of various factors, that of CD133 was decreased in the VHL transfectants and those of STAT3, JAK2 and Elongin A were eliminated. However, the expression of PTEN and of VHL was upregulated. These findings suggest that VHL regulated the
\end{abstract}

Correspondence to: Dr Hiroshi Kanno, Department of Neurosurgery, Yokohama City University School of Medicine, 3-9 Fukuura, Kanazawa-ku, Yokohama, Kanagawa 236-0004, Japan

E-mail: hiroshikannomd@nifty.com

Key words: glioma stem-like cells, von Hippel-Lindau protein, tumorigenicity, JAK/STAT pathway tumorigenicity and self-renewal ability of glioma cancer stem cells by inhibiting the JAK/STAT signaling pathway.

\section{Introduction}

Glioblastoma is one of the most intractable tumors of the central nervous system and is often fatal. Complete remission is rarely achieved. Compared to 30 years ago, the survival time has been prolonged only by a few months (1). Although chemotherapy, usually by oral temozolomide, may prolong survival of glioblastoma patients, it does not significantly affect survival. Bevacizumab, which is an anti-VEGF antibody, also extends survival time by approximately 2 months (2); it is not, however, curative. To date, the majority of glioblastoma patients succumb to the disease within 1 year of initial diagnosis. The therapeutic resistance of glioblastoma possibly results from the presence of cancer stem cells. The cancer stem cell theory does not apply to all types of cancer, but glioblastoma is considered a type of cancer possessing cancer stem/initiating cells (3), which have been isolated from the U87 human glioma cell line (4). The characteristics of glioma cancer stem cells include CD133 positivity, self-reproduction ability, neurosphere formation in non-serum medium containing growth factors, tumorigenicity in SCID mice, chemotherapy and radiotherapy resistance and cellular hierarchy. Signal transducer and activator of transcription 3 (STAT3) was reported to play a critical role in the tumorigenicity of glioma-initiating cells $(5,6)$. Inhibition of translation of STAT3 mRNA by STAT3 siRNA results in inhibition of cell proliferation and self-renewal (7). In addition, the expression of microRNA (miRNA)-21 in glioma-initiating cells is negatively regulated by activated STAT3 (8). It has been suggested that the JAK/STAT pathway is repressed by the von Hippel-Lindau (VHL) protein (9) and that BC-box proteins, including VHL, possibly inhibit the JAK/STAT pathway by binding to Elongin BC (10). Upregulated expression of PTEN coordinately inhibits the PI3K/Akt pathway (11) as well as VHL and PTEN function (12). Glioma stem-like cells (GSLCs) are suggested to be in a hypoxic environment (13). Furthermore, it was recently reported that TRAIL and paclitaxel synergize to kill U87-derived GSLCs in vitro (14) and that miRNA-34a suppresses cell proliferation and induces apop- 
tosis in U87-derived GSLCs. In this study, we demonstrated that VHL downregulated the tumorigenicity and self-renewal of U87-derived GSLCs by inhibiting the JAK/STAT signaling pathway and upregulated the expression of PTEN, which acted coordinately with VHL. We also analyzed the role of STAT3 in GSLCs and the possibility of therapy using VHL.

\section{Materials and methods}

Isolation of $\mathrm{CD} 133^{+}$U87-derived GSLCs. The human glioblastoma cell line U87MG was purchased from ATCC. U87MG is derived from a human glioblastoma and is widely used in biological studies on gliomas, particularly those examining the pathway involved in glioma proliferation. We initially cultured naive U87MG cells in Dulbecco's modified Eagle's medium (DMEM) containing 10\% fetal calf serum (FCS) (Fig. 1A) which was, according to a previous study (4), subsequently changed to non-serum DMEM/F12 containing B27 supplement (Gibco-Invitrogen, Grand Island, NY, USA), basic FGF (PeproTech EC Ltd., Rocky Hill, NJ, USA) and EGF (Upstate Biotechnology, Lake Placid, NY, USA). $\mathrm{CD}_{133^{+}}$cells were then selected by using an autoMACS ${ }^{\mathrm{TM}}$ Pro Separator (Miltenyi Biotec, Bergisch Gladbach, Germany) and neurosphere-forming cells were cultured in the same medium (Fig. 1B). Serial passage of the cells was performed by a mechanical dissociation method and passaged cells, i.e., GSLCs, were used for subsequent experiments.

Characterization of U87-derived GSLCs. Characterization of U87 GSLCs was carried out by immunocytochemistry using the following antibodies: anti-CD133 (Santa Cruz Biotechnology, San Diego, CA, USA), anti-STAT3 (Cell Signaling Technology, Danvers, MA, USA), anti-JAK2 (Santa Cruz Biotechnology), anti-VHL (Santa Cruz Biotechnology), anti-Elongin A (Abgent, San Diego, CA, USA), anti-PTEN (Santa Cruz Biotechnology), anti-NeuroD (Santa Cruz Biotechnology), anti-MAP2 (SigmaAldrich, St. Louis, MO, USA) and anti-GFAP antibodies (Dako, Glostrup, Denmark), as described below. In addition, cell proliferation, tumorigenicity in the subcutaneous tissue of SCID mice (Charles River, Yokohama, Japan), as well as soft agar colony and neurosphere formation were examined as described below.

Influence of VHL on U87-derived GSLCs. We hypothesized that STAT3 was inhibited by VHL, since most BC-box family proteins, including VHL, have the ability to inhibit the JAK/STAT pathway. STAT3 plays a role in stem cell maintenance. Therefore, we investigated whether VHL was able to downregulate STAT3 in GSLCs. Initially, we constructed a VHL-expressing adenovirus vector, as previously described (15).

VHL-expressing adenovirus vector. The VHL-expressing adenovirus vector was prepared as previously described (15). Adenovirus vector encoding human VHL (VHL54-213 amino acids) was generated by the use of the cosmid vector pAxCAwt. As a control vector, the vector for green fluorescent protein (GFP), generated by the use of pAxCAwt, was obtained from the Riken Gene Bank (Saitama, Japan). For adenovirus infection, U87 GSCs were seeded into 6-cm dishes $\left(1 \times 10^{6} \mathrm{cells} / \mathrm{cm}^{2}\right)$ 1 day prior to infection. The cells were then incubated for $1 \mathrm{~h}$ with $5 \mu$ of the virus solution diluted to $1 \times 10^{7}$ plaque-forming
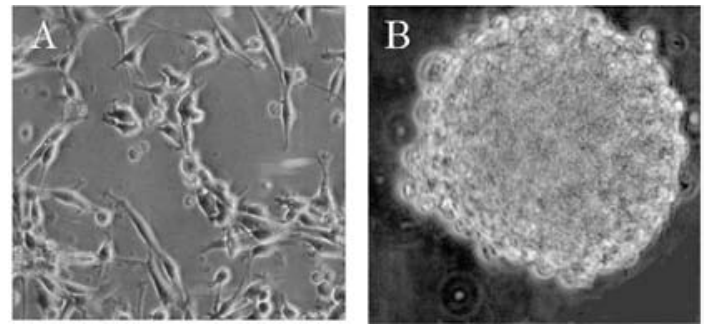

Figure 1. Morphology of cells. (A) Naive U87MG cells. (B) U87MG-derived glioma stem-like cells forming neurospheres.

units per milliliter in DMEM/F12 medium containing $5 \%$ FCS at a multiplicity of infection of 10 , which is a condition sufficient for nearly $100 \%$ cell infection. VHL-expressing adenovirus vector was transferred to U87 GSLCs following dissociation of the neurospheres into single cells. Two days following the transfection in dishes, some of the cultured cells were transferred onto a cover glass and subsequently fixed for immunocytochemical study while the remaining were used to extract protein for western blot analysis.

Fluorescence-immunocytochemical study using confocal laser microscopy. Naive U87MG cells attached to round cover slips were fixed for $24 \mathrm{~h}$ with $10 \%$ formalin naturally deodorized buffer solution. After irrigating twice with phosphate-buffered saline (PBS), the fixed cells were blocked with 5\% normal donkey serum for $30 \mathrm{~min}$. Subsequently, primary antibodies (anti-NeuroD, anti-MAP2, anti-STAT3, anti-VHL, anti-PTEN, anti-JAK2 and anti-GFAP) were diluted with PBS (1:200) and incubated with the cells for $1 \mathrm{~h}$ at room temperature. Following irrigation 3 times with PBS, the cells were incubated in the dark for $45 \mathrm{~min}$ at room temperature with FITC-linked anti-mouse IgG antibody (Sigma-Aldrich) or TRIC-linked anti-rabbit IgG antibody (Sigma-Aldrich) as secondary antibodies. Following a further 3 irrigations with PBS, the cells were incubated for 5 min with DAPI (Molecular Probes, Eugene, OR, USA) diluted 1:3,000 with PBS. Finally, the cover glasses bearing the cells were placed on glass slides following application of ProLong Gold Antifade Reagent (Life Technologies, Grand Island, NY, USA) to the slides. The cells were then observed under an FV300 confocal microscope (Olympus, Tokyo). Cell nuclei appeared as red fluorescence and primary antibodyreactive antigens as green fluorescence.

Western blot analysis. Protein was extracted from the cells with RIPA buffer (Thermo Scientific, Rockford, IL, USA; $0.05 \mathrm{M}$ Tris-HCl, pH 7.4, containing $0.15 \mathrm{M} \mathrm{NaCl}, 0.25 \%$ deoxycholic acid, $1 \%$ NP-40, $0.1 \%$ SDS, $1 \mathrm{mM}$ EDTA, $1 \mathrm{mM}$ PMSF, $1 \mathrm{mM}$ sodium orthovanadate, $1 \mathrm{mM}$ sodium fluoride, $1 \mu \mathrm{g} / \mathrm{ml}$ leupeptin and $1 \mu \mathrm{g} / \mathrm{ml}$ aprotinin). Extracted proteins were electrophoresed on 8-15\% SDS-PAGE gels and then transferred to polyvinylidene difluoride (PVDF) membranes by using an iBlot $^{\mathrm{TM}}$ Gel Transfer Device (Life Technologies) for $7 \mathrm{~min}$. The blots were probed with primary and secondary antibodies by using a SNAP id Protein Detection system (Merck Millipore, Darmstadt, Germany). Immunoreactive bands were visualized by chemiluminescence with ECL Western Blotting Detection Reagents (GE Healthcare, Japan). Images were analyzed with LAS-1000 (Fujifilm, Tokyo, Japan) and the density of the bands 
was determined by using Image Gauge software (Fujifilm). The following primary antibodies were used: anti-CD133 (Biorbyt, Cambridge, UK), anti-STAT3, anti-JAK2, anti-VHL and anti-PTEN. Horseradish peroxidase (HRP)-linked antirabbit IgG and HRP-linked anti-mouse IgG were used as the secondary antibodies.

Neurosphere formation assay. The neurosphere formation assay was performed as follows (16): neurosphere-forming U87 GSLCs were dissociated into single cells by continuous pipetting for $10 \mathrm{~min}$. Following confirmation of their single-cell status and dilution up to 5 cells $/ \mathrm{ml}, 200 \mu \mathrm{l}$ of the cell solution was placed into each well of a 96-well plate (mean, one cell per well). Subsequently, neurospheres $\geq 50 \mu \mathrm{m}$ in diameter in a single plate were counted under a phase-contrast microscope 1 week following placement of the cells.

Soft agar colony formation assay. This assay was performed as previously described (17). Briefly, following dissociation of the U87 GSLC neurospheres, a single-cell suspension $(1,000$ cells $/ \mathrm{ml})$ in $0.5 \mathrm{ml}$ of $0.3 \%$ agar in a medium consisting of $10 \% \mathrm{FCS}$ in DMEM/F12 was overlaid onto $0.5 \mathrm{ml}$ of $0.6 \%$ agar medium in the wells of 24 -well plates. Following 3 weeks of cultivation in a $5 \% \mathrm{CO}_{2}$ incubator, each well was examined under a stereoscopic microscope for colonies consisting of $>40$ cells.

Cell-proliferation assay. Cell proliferation was examined as previously described (18). After U87 GSLCs had been dissociated into single cells by $10-\mathrm{min}$ continuous pipetting using a long thin pipette, the cells were transfected with the VHL-expressing adenovirus vector or GFP-expressing adenovirus vector as a control. They were then cultured in the U87 GSLC maintenance medium. Starting 1 day after the cultures had been prepared, the number of cells was counted as follows: control vector-transfected and VHL-expressing vector-transfected U87 GSLCs were washed once with PBS, then dissociated into single cells by 10 -min continuous pipetting using a long thin pipette. The number of viable cells was counted following staining with trypan blue.

Implantation into SCID mice. Subcutaneous implantation of control- or VHL-transfectant U87 GSLCs $\left(1 \times 10^{4}\right.$ U87 GSLCs) into 4 SCID Hairless Outbred (SHO-Prkdc Hr) mice (Charles River) for each vector was performed. The mice were housed in a clean, temperature-controlled room on a 12-h day/night cycle with free access to food and water. One month following implantation, the mice were examined for subcutaneously-formed tumors. Formed tumors were histologically examined with hematoxylin-eosin staining and immunohistochemically with anti-CD133, anti-GFAP and anti-STAT3 antibodies. The animal experimental procedure was approved by the Institutional Animal Use Committee of Yokohama City University and was in accordance with the National Institutes of Health Guidelines for the care and use of laboratory animals.

Statistical analysis. Data were analyzed by ANOVA (SPSS II; SPSS, IBM, Tokyo, Japan) and a P-value of $<0.05$ was considered to indicate a statistically significant difference.

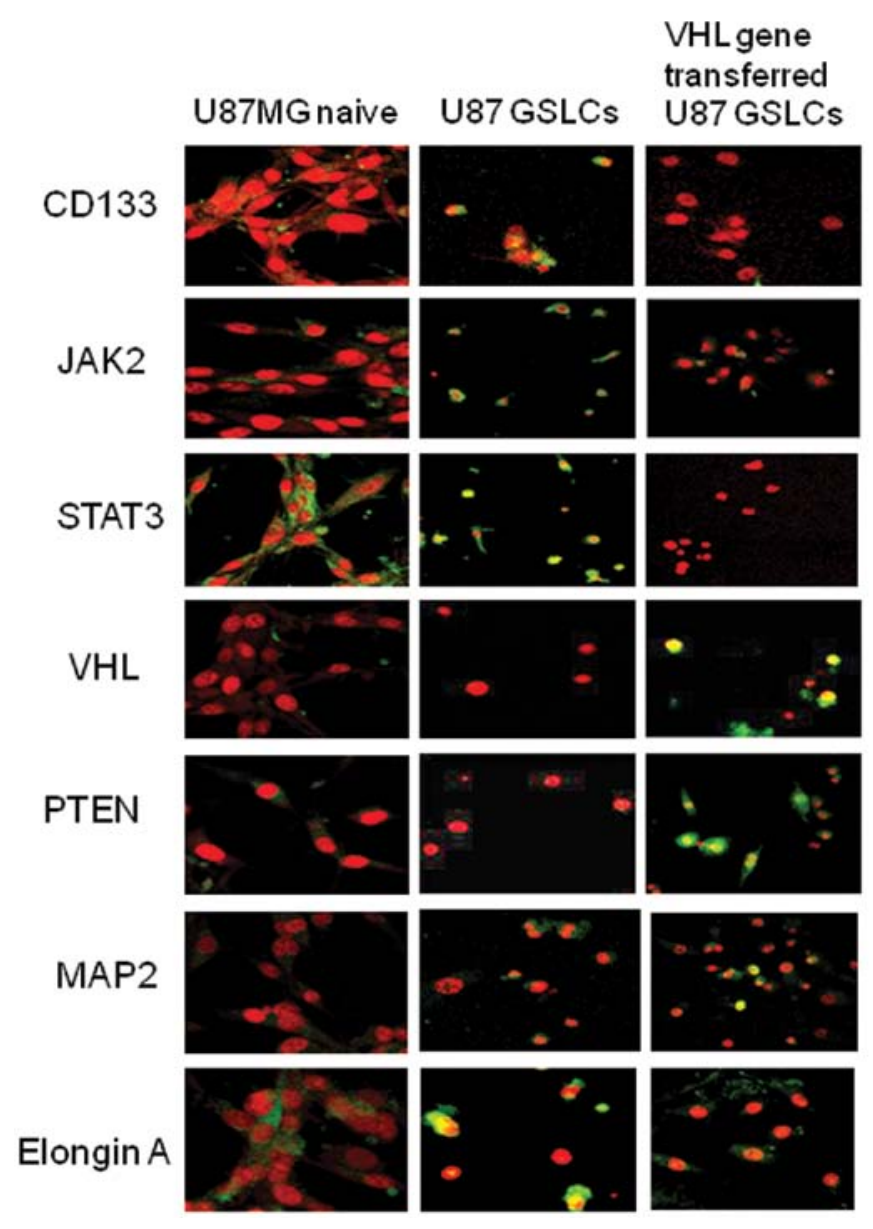

Figure 2. Fluorescence-immunocytochemical studies on U87MG naive cells, U87 glioma stem-like cells (GSLCs) and VHL-transfectant U87 GSLCs. Immunoreactivity toward primary antibodies is shown in green and DAPI-stained nuclei appears as red fluorescence.

\section{Results}

Immunocytochemical study on naive U87MG cells. Naive cells of the U87MG cell line were cultured as substrate-attached cells in DMEM containing 10\% FCS and the cells were passaged every 4-5 days. The cells displayed 2 or 3 cellular processes and were Elongin A positive, rarely CD133 positive, VHL negative and MAP2 negative. Also, the majority of the cells were STAT3 positive, partially JAK1 positive and PTEN negative.

U87MG-derived GSLCs. To obtain GSLCs from the U87MG cell line, we changed the medium to non-serum DMEM containing basic FGF, EGF and B27 supplement. One month later, the cultured cells tended to float. By using the MACS method with anti-CD133 antibody, we collected CD133positive fractioned cells, according to the manufacturer's instructions, and then cultured them in the above medium for 2-3 weeks. The cultured cells formed numerous floating neurospheres. The neurospheres were dissociated and some of the single cells were stained by fluorescence immunocytochemistry for characterization, whereas the remaining were used for soft agar colony and neurosphere formation, as well as for cell proliferation assays. In addition, protein was extracted from neurospheres for western blot analysis. 
A

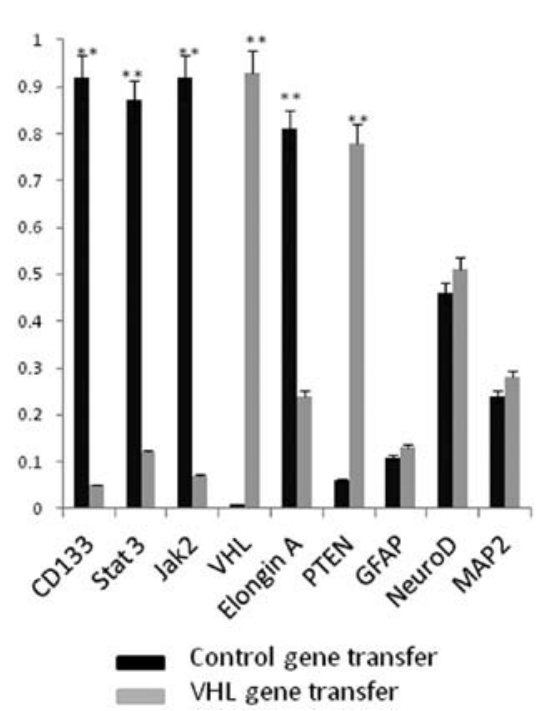

B

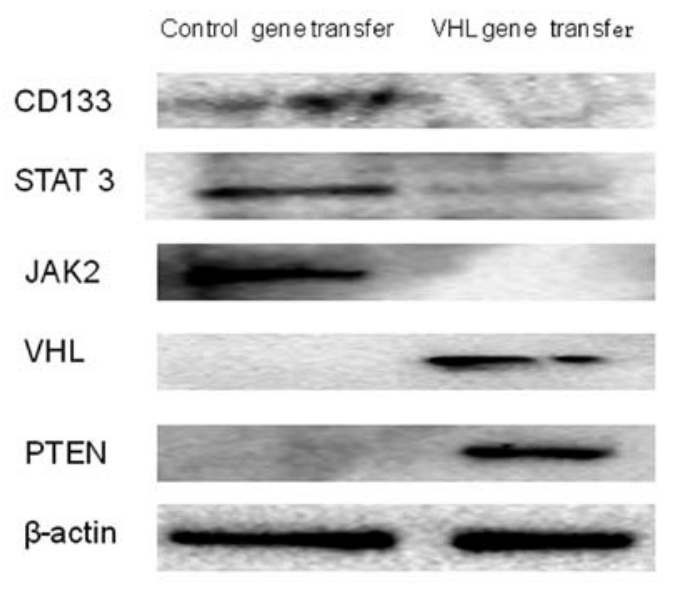

Figure 3. (A) Immunoreactivity rates for control-vector and VHL-vector glioma stem-like cell (GSLC) transfectants are shown. Expressions of CD133, STAT3, JAK2 and Elongin A were significantly inhibited, whereas those of VHL and PTEN were significantly increased in VHL-transfectant GSLCs compared to the control cells. Expressions of GFAP, NeuroD and MAP2 were not significantly altered. ${ }^{*} \mathrm{P}<0.05,{ }^{* *} \mathrm{P}<0.01$. (B) Western blot analysis of control-vector and VHL-vector GSLC transfectants. Control-vector GSLC transfectants expressed CD133, STAT3 and JAK2, whereas VHL-vector GSLC transfectants expressed VHL and PTEN, but exhibited inhibited expression of CD133, STAT3 and PTEN. $\beta$-actin expression was equal in both cells.

Fluorescence-immunocytochemical study on U87 GSLCs. Results of the immunocytochemical analysis of U87 GSLCs are depicted in Figs. 2 and 3A. The percentage of CD133positive cells as well as STAT3- and JAK2-positive cells was higher in the control-vector than in the VHL-vector transfectants. The majority of the naive U87 cells were Elongin A positive, as were the U87 GCLSs. U87 GSLCs were also VHL and PTEN negative, similar to U87 naive cells. Approximately half of those cells were NeuroD positive, of which some were GFAP positive.

Western blot analysis. The results of western blot analysis (Fig. 3B) supported the immunocytochemical data. U87 GSLCs expressed CD133, STAT3 and JAK2, but not VHL or PTEN. Expression of CD133, STAT3 and JAK1 was not detected following VHL gene transfer, contrary to VHL and PTEN expression, which was detected. Following VHL gene transfer to U87 GSLCs, the percentage of VHL and PTEN positive cells was increased, whereas that of CD133, STAT3 and JAK2 positive cells was decreased.

Soft agar colony formation, neurosphere formation and cell proliferation. The results of the colony-formation assay in soft agar medium demonstrated significantly greater colony formation in the control vector-transfected compared to the VHL-expressing vector-transfected U87 GSLCs $(\mathrm{P}<0.001)$ (Fig. 4A). Neurosphere formation, which is a reflection of the self-renewal ability, was also significantly greater in the former than in the latter $(\mathrm{P}<0.001)$ (Fig. 4B). Proliferation of control vector-transfected U87 GSLCs was significantly more pronounced compared to the VHL gene-transfected GSLCs 7 days following transfection $(\mathrm{P}<0.01)$ (Fig. $4 \mathrm{C})$, although the difference between them was smaller than in the case of the soft agar colony or neurosphere formation assay.
Implantation of U87 GSLCs into SCID mice. Although subcutaneous transplantation of U87 GSLCs into SCID mice always resulted in tumor formation, transplantation of VHL gene-transfected U87 GSLCs resulted in markedly reduced or no tumor formation. The tumors arising from the U87 GSLCs transplanted into the SCID mice exhibited the pathological features of glioblastoma and most of the cells in the tumor tissue were CD133- and STAT3- positive but showed no immunoreactivity indicative of GFAP (Fig. 5).

\section{Discussion}

Glioma cancer stem cells are defined by their self-renewal ability, CD133-positivity, and transplantation ability in SCID mice (3). U87 GSLCs constructed by us exhibited these properties and may thus be considered a subpopulation of naive U87 cells. U87 GSLCs may be cultured in non-serum medium containing growth factors such as basic FGF and EGF. Glioma cancer stem cells are also known as glioma-initiating cells. GSLCs possess various mechanisms related to treatment tolerability, epigenetics and PTEN/PI3K/Akt signaling (19), and they reside in a hypoxic niche (14). Our results suggest that U87 GSLCs had a high capacity for colony and neurosphere formation. VHL inhibited STAT3, JAK2 and Elongin A. In addition, VHL upregulated PTEN expression. However, GFAP, NeuroD and MAP2 levels were not significantly affected by the VHL gene transfer. These results suggest that VHL affected the JAK/STAT pathway as well as the PTEN/PI3K/Akt pathway; they also suggest that upregulation of PTEN by VHL gene transfer may affect the PI3K/Akt pathway, since PTEN is a PI3K/Akt pathway inhibitor (19). STAT3 has been reported to play an important role in the self-renewal ability of cancer stem cells. VHL inhibited the implantation ability, as well as soft agar colony and neurosphere formation, all of which are 

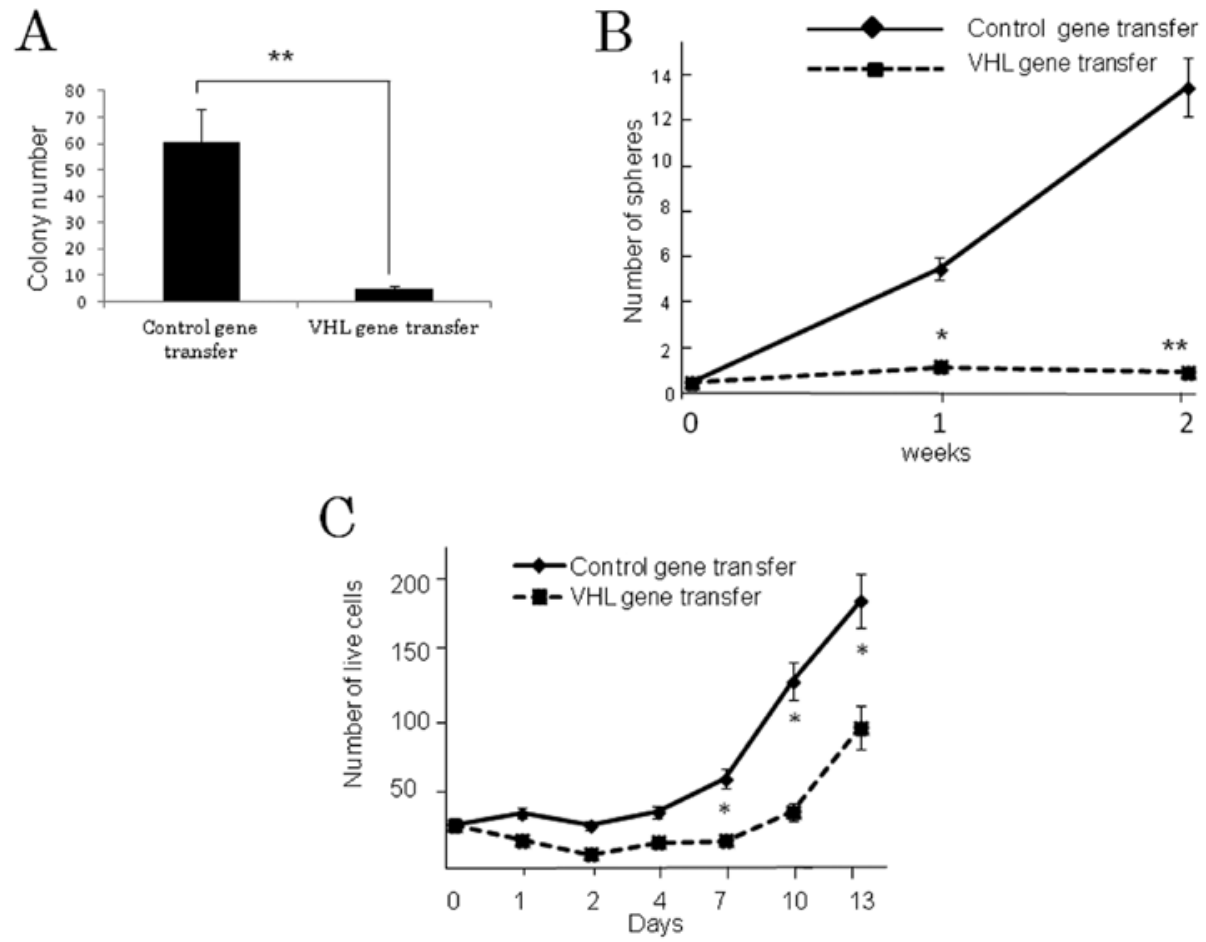

Figure 4. (A) Results on soft agar colony formation by control-vector and VHL-vector GSLC transfectants. Colony formation by the VHL transfectants was significantly inhibited. (B) Results for neurosphere formation. After one week, soft agar colony formation of VHL gene-transfected GSLCs was significantly inhibited. (C) Results of cell-proliferation assay. After 7 days, control gene-transfected GSLCs showed significantly greater proliferation than the VHL genetransfected GSLCs $(\mathrm{P}<0.01) .{ }^{*} \mathrm{P}<0.01,{ }^{* *} \mathrm{P}<0.001$.
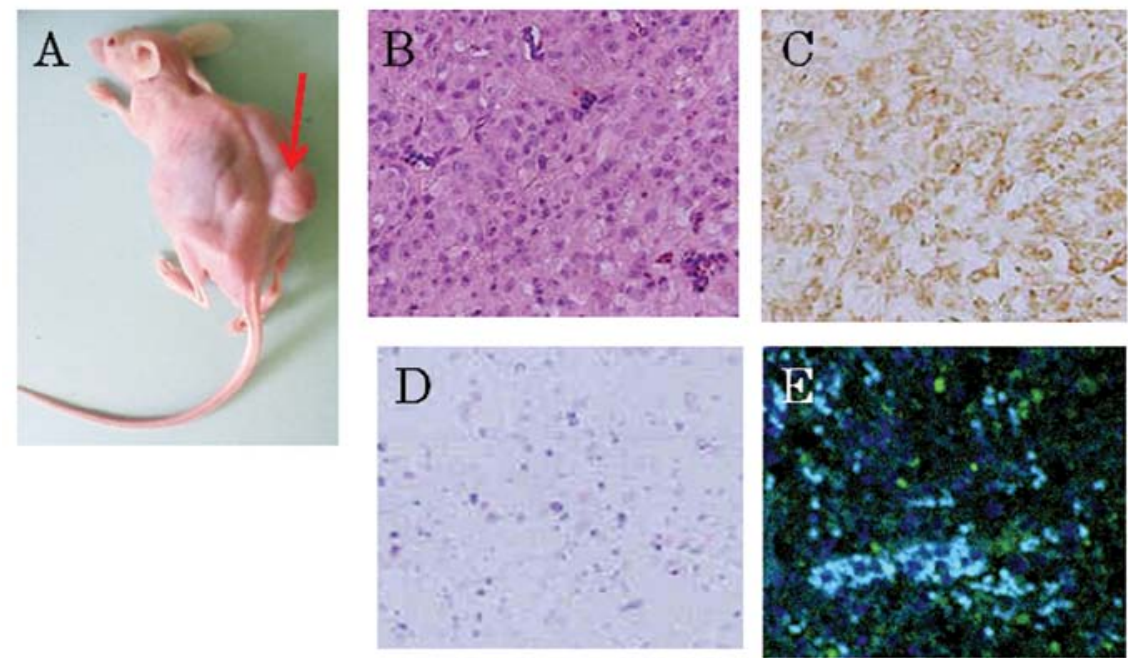

Figure 5. (A) Subcutaneously implanted GSLCs in the SCID mouse (red arrow). (B) Hematoxylin-eosin staining of subcutaneously implanted GSCs. Histologically, the appearance was similar to human glioblastomas. (C) Immunostaining by the Avidin-Biotin Complex (ABC) method for the detection of STAT3. Cytoplasm of most cells shows immunoreactivity toward anti-STAT3 antibody. Counter-staining with hematoxylin. (D) Immunostaining by the ABC method for examination of GFAP expression. Only few cells are immunoreactive with anti-GFAP antibody. Counter-staining with hematoxylin. (E) Fluorescence immunostaining for detection of CD133 expression. The majority of the cells are immunopositive for CD133 (green). Nuclei are stained with DAPI (light blue).

key characteristics of cancer stem cells. Our results suggest that these characteristics may be related to the JAK/STAT or PTEN/PI3K/Akt pathways in GSLCs. VHL inhibits HIF-1 $\alpha$ under normoxic, but not under hypoxic, conditions by acting through the ubiquitin/proteasome system (20). Although $V H L$ gene mutations are not frequently found in gliomas (21), function of the $V H L$ gene is maintained under normoxic, but not hypoxic, conditions (20). In addition, overexpression of VHL inhibits tumorigenesis and reduces the proliferation of glioma cells (22). The core of glioblastomas is reported to be in the hypoxic state and glioblastomas are characterized by their necrotic regions due to poor vascularization, which leads to inadequate blood supply and, consequently, to hypoxic and necrotic areas (23). It is suggested that GSLCs are maintained 
in vivo in a niche characterized by reduced oxygen tension, i.e., in a hypoxic niche (13). U87-derived GCLSs exhibited negative expression of the VHL protein and, thus, the use of U87 GCLSs may be considered adequate for characterization of GCLSs. Overexpression of VHL upregulated PTEN and downregulated the JAK/STAT pathway, although it did not significantly affect the expression of neuronal differentiation markers such as NeuroD, GFAP and MAP2. In addition, overexpression of VHL significantly downregulated the proliferation of U87 GSLCs. However, the overexpression of VHL inhibited cell proliferation of U87 GSLCs to a lesser extent than it did soft agar colony and neurosphere formation, or implantation capacity into SCID mice. These results suggest that VHL inhibited the tumorigenicity and selfrenewal ability via the JAK/STAT pathway and also affected the PTEN/PI3K/Akt pathway, both of which are critical in GSLCs, but it did not affect the differentiation of the GSLCs.

In conclusion, VHL overexpression downregulated the tumorigenicity and self-renewal ability in U87 GCLSs via the JAK/STAT pathway and upregulated PTEN. VHL overexpression therapy may be promising for the regulation of GSLCs under hypoxic conditions.

\section{Acknowledgements}

This study was supported by a grant from the Education and Science Ministry of Japan [Basic Research (B) 23390353]. The authors thank Ms. Akemi Miura for her technical assistance.

\section{References}

1. Stupp R, Mason WP, van den Bent MJ, et al: Radiotherapy plus concomitant and adjuvant temozolomide for glioblastoma. N Engl J Med 352: 987-996, 2005.

2. Friedman HS, Prados MD, Wen PY, et al: Bevacizumab alone and in combination with irinotecan in recurrent glioblastoma. J Clin Oncol 27: 4733-4740, 2009.

3. Singh SK, Hawkins C, Clarke ID, Squire JA, Bayani J, Hide T, Henkelman RM, Cusimano MD and Dirks PB: Identification of human brain tumour initiating cells. Nature 432: 396-401, 2004.

4. Yu SC, Ping YF, Yi L, Zhou ZH, Chen JH, Yao XH, Gao L, Wang JM and Bian XW: Isolation and characterization of cancer stem cells from a human glioblastoma cell line U87. Cancer Lett 265: 124-134, 2008.

5. Sherry MM, Reeves A, Wu JK and Cochran BH: STAT3 is required for proliferation and maintenance of multipotency in glioblastoma stem cells. Stem Cells 27: 2383-2392, 2009.

6. Villalva C, Martin-Lannerée S, Cortes U, Dkhissi F, Wager M, Le Corf A, Tourani JM, Dusanter-Fourt I, Turhan AG and Karayan-Tapon L: STAT3 is essential for the maintenance of neurosphere-initiating tumor cells in patients with glioblastomas: a potential for targeted therapy? Int J Cancer 128: 826-838, 2011.

7. Yang YP, Chang YL, Huang PI, et al: Resveratrol suppresses tumorigenicity and enhances radiosensitivity in primary glioblastoma tumor initiating cells by inhibiting the STAT3 axis. J Cell Physiol 227: 976-993, 2012.
8. Ohno M, Natsume A, Kondo Y, Iwamizu H, Motomura K, Toda $\mathrm{H}$, Ito $\mathrm{M}$, Kato $\mathrm{T}$ and Wakabayashi T: The modulation of microRNAs by type I IFN through the activation of signal transducers and activators of transcription 3 in human glioma. Mol Cancer Res 7: 2022-2030, 2009.

9. Ivanov SV, Salnikow K, Ivanova AV, Bai L and Lerman MI: Hypoxic repression of STAT1 and its downstream genes by a pVHL/HIF-1 target DEC1/STRA13. Oncogene 26: 802-812, 2007.

10. Kamura T, Sato S, Haque D, Liu L, Kaelin WG Jr, Conaway RC and Conaway JW: The Elongin BC complex interacts with the conserved SOCS-box motif present in members of the SOCS, ras, WD-40 repeat, and ankyrin repeat families. Genes Dev 12: 3872-3881, 1998

11. Dasari VR, Kaur K, Velpula KK, Gujrati M, Fassett D, Klopfenstein JD, Dinh DH and Rao JS: Upregulation of PTEN in glioma cells by cord blood mesenchymal stem cells inhibits migration via downregulation of the PI3K/Akt pathway. PLoS One 5: e10350, 2010

12. Frew IJ, Thoma CR, Georgiev S, Minola A, Hitz M, Montani M, Moch H and Krek W: pVHL and PTEN tumour suppressor proteins cooperatively suppress kidney cyst formation. EMBO J 27: 1747-1757, 2008.

13. Bar EE: Glioblastoma, cancer stem cells and hypoxia. Brain Pathol 21: 119-129, 2011.

14. Qiu B, Sun X, Zhang D, Wang Y, Tao J and Ou S: TRAIL and paclitaxel synergize to kill U87 cells and U87-derived stem-like cells in vitro. Int J Mol Sci 13: 9142-9156, 2012.

15. Yamada H, Dezawa M, Shimazu S, Baba M, Sawada H, Kuroiwa Y, Yamamoto I and Kanno H: Transfer of the von Hippel-Lindau tumor suppressor gene to neuronal progenitor cells in treatment for Parkinson's disease. Ann Neurol 54: 352-359, 2003.

16. Hägerstrand D, He X, Bradic Lindh M, Hoefs S, Hesselager G, Ostman A and Nistér M: Identification of a SOX2-dependent subset of tumor- and sphere-forming glioblastoma cells with a distinct tyrosine kinase inhibitor sensitivity profile. Neuro Oncol 13: 1178-1191, 2011.

17. Kanno H, Kuwabara T, Shinonaga M, Chang CC, Tanaka Y, Sugio Y, Morita H, Yasumitsu H, Umeda M and Nagashima Y: Establishiment of a human glioma cell line bearing a homogeneously staining chromosomal region and releasing $\alpha$ - and $\beta$-type transforming growth factors. Acta Neuropathol 79: 30-36, 1989.

18. Yamada S, Kanno H and Kawahara N: Trans-membrane peptide therapy for malignant glioma by use of a peptide derived from the MDM2 binding site of p53. J Neurooncol 109: 7-14, 2012.

19. Bleau AM, Hambardzumyan D, Ozawa T, Fomchenko EI, Huse JT, Brennan CW and Holland EC: PTEN/PI3K/Akt pathway regulates the side population phenotype and ABCG2 activity in glioma tumor stem-like cells. Cell Stem Cell 4: 226-235, 2009.

20. Maxwell PH, Wiesener MS, Chang GW, Clifford SC, Vaux EC, Cockman ME, Wykoff CC, Pugh CW, Maher ER and Ratcliffe PJ: The tumour suppressor protein VHL targets hypoxia-inducible factors for oxygen-dependent proteolysis. Nature 399: 271-275, 1999.

21. Kanno H, Shuin T, Kondo K, Yamamoto I, Ito S, Shinonaga M, Yoshida $M$ and Yao $M$ : Somatic mutations of the von Hippel-Lindau tumor suppressor gene and loss of heterozygosity on chromosome $3 p$ in human glial tumors. Cancer Res 57: 1035-1038, 1997.

22. Sun X, Liu M, Wei Y, Liu F, Zhi X, Xu R and Krissansen GW: Overexpression of von Hippel-Lindau tumor suppressor protein and antisense HIF-1alpha eradicates gliomas. Cancer Gene Ther 13: 428-435, 2006.

23. Valk PE, Mathis CA, Prados MD, Gilbert JC and Budinger TF: Hypoxia in human gliomas: demonstration by PET with fluorine18-fluoromisonidazole. J Nucl Med 33: 2133-2137, 1992. 